\title{
Factors affecting the impact of olfactory loss on the quality of life and emotional coping ability*
}

\author{
Chih-Hung Shu ${ }^{1}$, Po-Lei Lee ${ }^{2}$, Ming-Ying Lan ${ }^{1}$, Yi-Lun Lee ${ }^{1}$ \\ 1 Department of Otolaryngology, Taipei Veterans General Hospital and National Yang-Ming \\ University School of Medicine, Taipei, Taiwan \\ 2 Department of Electrical Engineering, National Central University, Jhongli, Taiwan
}

\begin{abstract}
SUMMARY Background: Olfactory impaired patients have decreased quality of life and may need to develop a coping ability for the olfactory loss. This study investigated how factors like olfactory function, disease duration, etiology, age, and gender affect patients' quality of life and emotional ability to cope.

Methods: Four hundred and thirteen consecutive patients with the chief complaint of olfactory dysfunction were evaluated. The Questionnaire of Olfactory Disorders (QOD) included negative statements $(Q O D-N S)$ that indicated the impact on the quality of life, and positive statements (QOD-PS) reflecting the emotional coping ability. Relations between studied factors and QOD-NS or QOD-PS were analyzed.

Results: Poorer olfaction and younger age correlated with increased QOD-NS scores, whereas longer disease duration and older age correlated with increased QOD-PS scores. Females had poorer coping than males. QOD-PS scores were inversely related to QOD-NS scores. Conclusions: The impact of olfactory loss is more significantly felt by younger patients with poorer olfaction. Older patients or those with longer disease duration develop better emotional coping abilities so as to reduce the impact on quality of life. It may be helpful for the patients with olfactory loss to develop emotional coping as early as possible to decrease the olfactory impact.
\end{abstract}

Key words: quality of life, smell, olfaction disorders, questionnaire, coping skills

\section{INTRODUCTION}

Olfactory function is important for tasting food and detecting environmental dangers. Daily life activities like eating, cooking, detecting gas leak or smoke, and personal hygiene are the most frequently cited impairments in olfactory dysfunction ${ }^{(1-7)}$. Olfactory impaired patients have more difficulties in daily life activities and decreased quality of life, which are influenced by certain factors. For example, more severe olfactory loss correlates with increased complaint score and rating of impaired quality of life ${ }^{(1-4,7)}$, younger patients have more difficulties in daily life ${ }^{(2)}$, and females have more complaints and suffer more than males ${ }^{(2,3)}$. Etiology and duration of olfactory dysfunction do not have significantly different effects on complaint score or quality of life $\mathrm{e}^{(2,3)}$.

Prognosis is often not satisfactory, with only $10 \%$ and $32 \%$ of patients with post-traumatic or post-URI olfactory impairment, respectively, achieving improved olfactory function ${ }^{(8)}$.
In most cases, olfactory function is not restored to normal agerelated level ${ }^{(9)}$. Patients may develop emotion- or problemfocused strategies to cope ${ }^{(10)}$. However, factors that influence the coping ability are not well investigated, with age as the only reported factor associated with coping ability ${ }^{(3)}$.

Evaluating the quality of life through a questionnaire can cover key points under study. The Questionnaire of Olfactory Disorders (QOD) is an instrument specifically designed to evaluate the quality of life of patients with olfactory dysfunction ${ }^{(3)}$. It includes negative statements (QOD-NS) that indicate impairments in the quality of life that patients suffer, and positive statements (QOD-PS) that indicate the level of emotional ability to cope ${ }^{(3)}$. This study aimed to determine the impact of factors like disease duration, self-rated olfaction, etiology, age, and gender on QOD-NS and QOD-PS in patients with olfactory loss. 


\section{MATERIALS AND METHODS \\ Patients}

Four hundred and thirteen consecutive patients aged $13-85$ years (mean, $46.3 \pm 15.3$ years) with a chief complaint of olfactory dysfunction were enrolled. The male to female ratio was 0.94:1. Detailed histories about the duration, course, and etiology of the olfactory dysfunction, as well as the accompanying nasal symptoms, were obtained. The Institutional Review Board of Taipei Veterans General Hospital approved the study, which was performed in accordance with the declaration of Helsinki on Biomedical Research involving human subjects. Nasal endoscopic examination was performed in all patients to determine any sino-nasal diseases, with emphasis on the region of the olfactory cleft and middle meatus. The etiology of olfactory loss was determined by events related to the olfactory disorders, the course of dysfunction, and related medical or surgical diseases. Before the olfactory test, patients were asked to rate their olfactory function as 'complete loss', 'poorer than normal', or 'normal,' and in a visual analogue scale (VAS), from ' 0 ' as complete loss of olfactory function to ' 10 ' as extremely sensitive olfactory function.

\section{Olfactory test}

Olfactory function was assessed with the 'Sniffin' Sticks' olfactory test, which consisted of odour threshold (T), odour discrimination (D), and odour identification (I) tasks, which were performed as previously reported ${ }^{(11)}$. A portion of the descriptors in the odour identification were modified to more familiar ones in Taiwan ${ }^{(12)}$. Scores of 'T' (range, 1 - 16), 'D' (range, 0 - 16), and 'I' (range, 0 - 16) were summed-up as the TDI score (range, 1-48), with A TDI score $\leq 15$ diagnosed as anosmia, $\geq$ 30 as normosmia, and a score in between as hyposmia ${ }^{(13)}$.

\section{Questionnaire for quality of life}

The 'Dresden Questionnaire for Olfactory Disorders' (QOD) consisted of 19 statements grouped into two domains: 17 'negative' statements (QOD-NS) and 2 'positive' statements (QOD-PS) ${ }^{(3)}$. Patients checked 'I agree,' 'I agree partly,' 'I disagree partly,' or 'I disagree' for each statement.

Table 1. Summary of olfactory impact on quality of life and emotional ability to cope.

\begin{tabular}{lcc}
\hline Factors & $\begin{array}{c}\text { Impact on quality } \\
\text { of life }\end{array}$ & $\begin{array}{c}\text { Emotional ability to } \\
\text { cope with olfactory } \\
\text { loss }\end{array}$ \\
\hline $\begin{array}{l}\text { Lower self-rated } \\
\text { olfactory function }\end{array}$ & Increasing & Not correlated \\
$\begin{array}{l}\text { Lower measured } \\
\text { olfactory function }\end{array}$ & Increasing & Not correlated \\
$\begin{array}{l}\text { Longer disease } \\
\text { duration }\end{array}$ & Not correlated & Increasing \\
$\begin{array}{l}\text { Age older than } 30 \\
\text { Gender }\end{array}$ & Decreasing & Increasing \\
Etiology & Non-significant & Poorer in female \\
\hline
\end{tabular}

The QOD-NS indicated impaired quality of life the patients experienced, with 3 points assigned for checking 'I agree,' 2 for 'I agree partly,' 1 for 'I disagree partly,' and 0 for 'I disagree.' Higher scores indicated more severe impairment.

The QOD-PS statements were 'I can imagine adjusting my difficulties with smelling' and 'I have to learn to live with my difficulties with smelling,' which indicated the level of emotional coping with the olfactory impairment ${ }^{(10)}$. Assignment of scores was the same as those in the QOD-NS, with higher QOD-PS scores indicating improved ability to cope. Since QOD-NS and QOD-PS indicated different domains, they were analyzed separately with different factors.

\section{Statistical analysis}

A Spearman correlation was used to analyze self-rated olfaction with QOD-NS score, while the Kruskal-Wallis test was used to analyze QOD-PS score in the three self-rated olfaction groups. Pearson correlation was used to correlate TDI or disease duration with QOD-NS or QOD-PS scores. Multiple linear regression was used to analyze the four major etiology groups (i.e., post-traumatic, post-viral, sino-nasal, and idiopathic) with QOD-NS or QOD-PS scores.

Two-Sample t-test was used to analyze the effects of gender on QOD-NS and QOD-PS scores. Linear regression was used to correlate QOD-NS and QOD-PS scores with age, and with each other (between QOD-PS and QOD-NS scores). A p < 0.05 was considered statistically significant.

\section{RESULTS}

Olfactory dysfunction and age influenced impairment of quality of life

There was greater impact on quality of life associated with poor self-rated olfaction, expressed by the significant but inverse correlation between self-rated olfaction with VAS and QOD-NS score (Spearman correlation, $r=-0.300, p<0.001$ ) (Table 1).

The TDI scores correlated significantly with the self-rated olfactory function with VAS (Spearman correlation, $r=0.655$; $\mathrm{p}<0.001$ ). Similarly, the TDI scores correlated inversely with QOD-NS scores (Pearson correlation, $r=-0.211, p<0.001$; Table 1), which indicated that lower measured olfactory function meant significantly greater impact on the quality of life.

The QOD-NS score changed significantly with age up to 33 years (linear regression, $y=24.527+0.875 x ; y=$ QOD-NS score, $x=$ age; $r=0.235, p=0.021$ ) (Figure 1A), but changed inversely with age $\geq 34$ years (linear regression, $y=65.142$ - $0.413 \mathrm{x} ; \mathrm{y}=$ QOD-NS score, $\mathrm{x}=$ age; $\mathrm{r}=0.190, \mathrm{p}=0.001$ ) (Figure 1B), indicating that increasing age up to 33 years correlated with greater olfactory impact on life quality but with less impact for 33 years and older.

In terms of etiology, $125(30.3 \%)$ patients were post-traumatic, $103(24.9 \%)$ sino-nasal, 64 (15.5\%) post-viral, and $95(23.0 \%)$ idiopathic. The QOD-NS scores did not change significantly with different etiologies of olfactory dysfunction after adjust- 
(A)

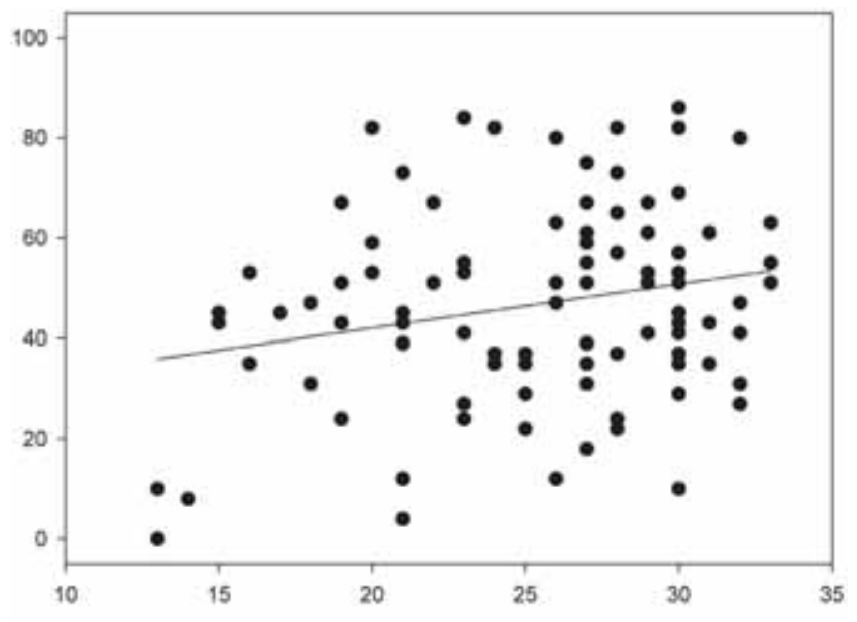

(B)

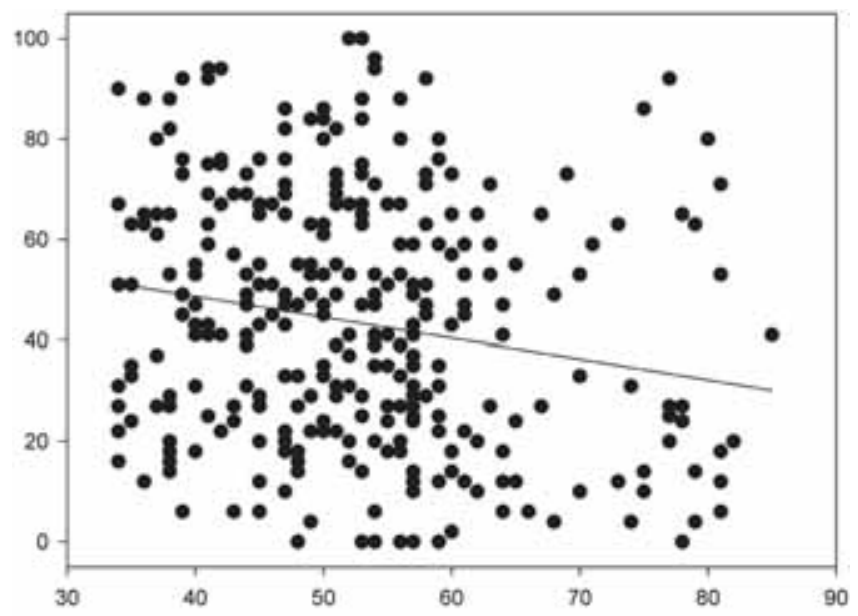

Figure 1. Correlation of QOD-NS score and age. (A) The QOD-NS score changed significantly with age $\leq 33$ years (linear regression, $\mathrm{y}=24.527+0.875 \mathrm{x}$; $y=$ QOD-NS score, $x=$ age; $r=0.235, p=0.021$ ). (B) The QOD-NS score changed significantly and inversely with age $\geq 34$ years (linear regression, $\mathrm{y}=65.142-0.413 \mathrm{x} ; \mathrm{y}=$ QOD-NS score, $\mathrm{x}=$ age; $\mathrm{r}=0.190, \mathrm{p}=0.001)$.

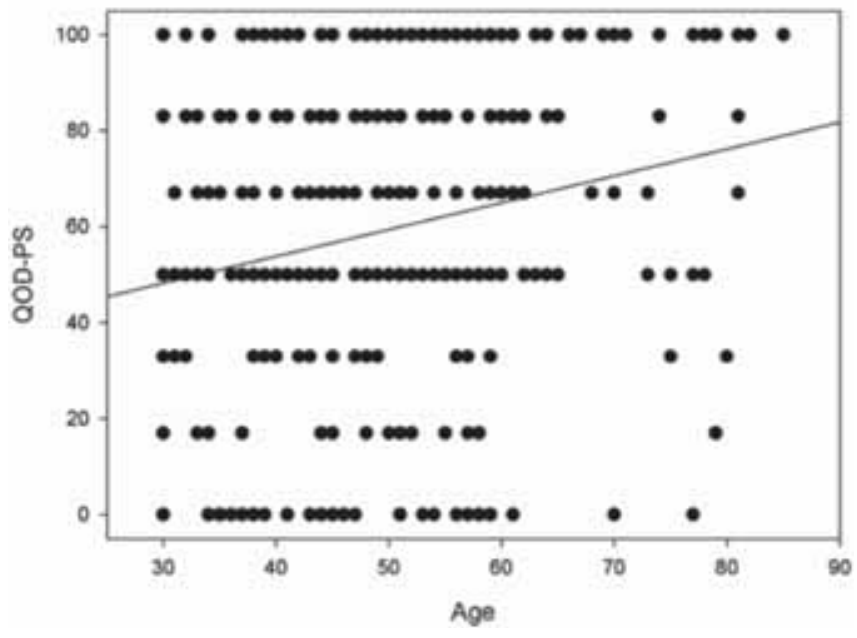

Figure 2. Correlation of QOD-PS score and age. The QOD-PS score changed with age $\geq 30$ years (linear regression, $y=31.501+0.559 x ; y=$ QOD-PS score, $\mathrm{x}=$ age; $\mathrm{r}=0.209, \mathrm{p}<0.001$ ).

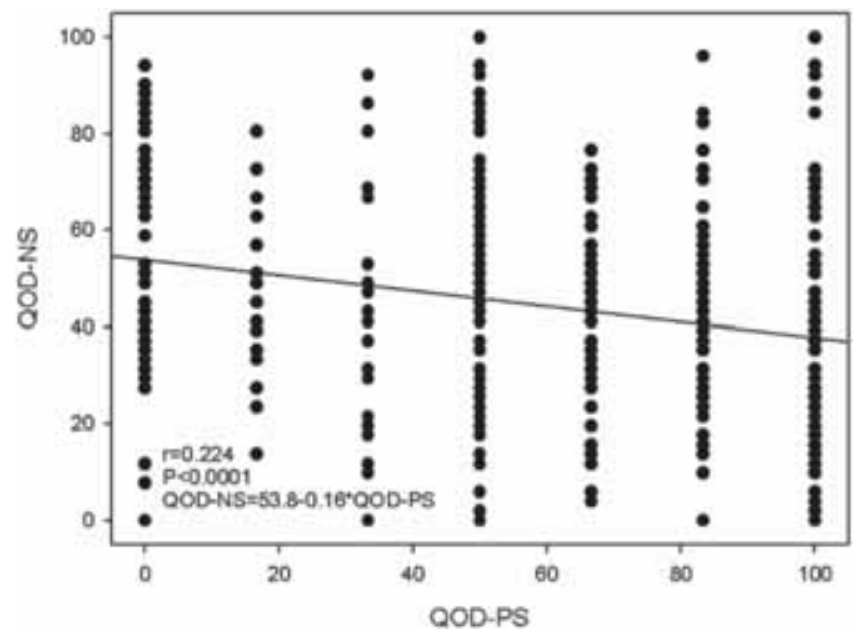

Figure 3. Correlation of QOD-PS and QOD-NS scores. The QOD-PS score correlated significantly with QOD-NS score (linear regression, $\mathrm{y}=53.8$ $0.16 \mathrm{x} ; \mathrm{y}=$ QOD-NS score, $\mathrm{x}=$ QOD-PS score; $\mathrm{r}=0.224, \mathrm{p}<0.001$ ).

(linear regression, $\mathrm{y}=31.501+0.559 \mathrm{x} ; \mathrm{y}=$ QOD-PS score, $\mathrm{x}=$ age; $\mathrm{r}=0.209, \mathrm{p}<0.001$; Figure 2), but not with age $<30$ years, which indicated improved coping ability after the age of 30 years. Females had poorer emotional coping than males, based on the lower QOD-PS scores in females (56.62 \pm 33.59$)$ than in males $(63.15 \pm 31.98)(\mathrm{p}=0.044$, by $\mathrm{t}$-test; Table 1$)$.

However, the QOD-PS score was not related with self-rated olfaction ( $p=0.41$, by Kruskal-Wallis test; Table 1), measured olfactory function $(\mathrm{r}=-0.096, \mathrm{p}=0.059$, by Pearson correlation), or etiology ( $\mathrm{p}$ etiology $=0.136$, by multiple linear regression; Table 1).

Increased emotional ability to cope correlated with decreased olfactory impact on quality of life

The QOD-PS scores inversely correlated with QOD-NS 
scores (linear regression, $\mathrm{y}=53.8-0.16 \mathrm{x} ; \mathrm{y}=$ QOD-NS score, $\mathrm{x}=$ QOD-PS score; $\mathrm{r}=0.224, \mathrm{p}<0.001$; Figure 3), indicating that increased emotional ability to cope decreased the olfactory impact on quality of life.

\section{DISCUSSION}

The results in the current manuscript are consistent with previous reports, where self-rated olfaction ${ }^{(1)}$, measured olfactory function, and age (2) are associated with olfactory impact on quality of life. However, unlike in previous reports, gender shows no significant impact (Table 1) ${ }^{(3)}$. Disease duration, age (3), and gender reveal different effects of the emotional ability to cope with olfactory dysfunction (Table 1).

Self-rated olfactory function reflects subjective feeling regarding olfactory function, while quality of life is an assessment of the subjective opinion on satisfaction in life. As such, selfrated olfaction may reflect the olfactory impact on quality of life. The results here reveal that poorer self-rated olfaction has greater impact on self-rated quality of life (Table 1). Olfactory impaired patients with improved self-rated olfactory function have less disability in daily living than subjectively and persistently impaired patients ${ }^{(1)}$. Furthermore, $87 \%$ patients with subjectively improved olfaction report satisfaction with life, significantly higher than the $50 \%$ in persistently impaired patients ${ }^{(1)}$. Thus, self-rated olfaction consistently correlates well with olfactory impact on quality of life.

In this study, self-rated olfactory function correlates well with TDI score ( $r=0.655 ; \mathrm{p}<0.001$, by Spearman correlation). Although patients may inaccurately rate their olfactory function ${ }^{(14)}$, the precision of self-rating increases if people are trained or if attention is given to the sense of smell in daily life (15). Patients with chief complaint of olfactory impairment indicate that they sensed changes in their olfactory function. Thus, a correlation between self-rated and measured olfactory function may be valid in such patients ${ }^{(1,4)}$.

By extension, it is reasonable to infer that measured olfactory function, similar to self-rated olfaction, may also correlate with quality of life. Using the Questionnaire of Olfactory Dysfunction, normosmic patients have significantly less impact on quality of life than hyposmic or anosmic patients, and TDI scores significantly correlate with QOD-NS scores ${ }^{(3)}$. These corroborate results where poorer measured olfactory function meant greater impact on quality of life (Table 1).

Disease duration is not significantly correlated with impact on life quality (Pearson correlation with QOD-NS, $r=-0.014$, $\mathrm{p}=0.796$, Table 1). Frasnelli et al. also report that quality of life does not correlate significantly with disease duration ${ }^{(3)}$, while Temmel et al. have shown that subjective decreases in quality of life are not significantly different in three duration groups, i.e., $<24$ months, $24-48$ months and $>48$ months (2). Furthermore, disease duration does not have a differential effect on quality of life, which is consistent in this and in previous reports ${ }^{(2,3)}$. Nevertheless, it may take time for olfac- tory function to recover, or it may never be fully restored ${ }^{(8,9)}$. Patients may adopt multiple coping strategies over time ${ }^{(10)}$ and thus, those with longer disease duration have better chances of developing emotional coping for olfactory loss $(\mathrm{p}<0.001$, by Pearson correlation; Table 1).

Age is an important factor for both olfactory impact on quality of life and emotional coping ability for olfactory loss (Figures 1 and 2). The results here reveal that olfactory impact on quality of life increases with age up to 33 years (Figure 1A), while older age correlates with decreasing impact (Figure 1B) and increasing emotional coping ability (Figure 2). Temmel et al. (2) have found significant differences in complaint score in three age groups (i.e., $54 \%$ in < 41 years, $53 \%$ in $41-60$ years, and $38 \%$ in $>60$ years). The degree of difficulties in daily life is highest in the youngest group, and decreases with age ${ }^{(2)}$. Emotional coping ability for olfactory loss also correlates significantly with age in previous reports ${ }^{(3)}$. From the viewpoint of age, there is consistency in that improved emotional coping ability is accompanied by less olfactory impact on quality of life in previous reports ${ }^{(2,3)}$ and in this study (Figures 1B and 2). Thus, by analyzing the correlation of QOD-NS with QODPS scores, emotional coping ability correlates significantly with decreasing olfactory impact on quality of life (linear regression, $r=0.224, p<0.001$; Figure 3 ). Patients younger than 30 years should be especially noted for their increasing olfactory impact but without the concomitant increase in emotional coping ability.

The two queries in the QOD, 'I visit friends, relatives, or neighbours less often' and 'I avoid groups of people,' which may be regarded as problem-focused coping strategies ${ }^{(10)}$ are not included in the QOD-PS used here. However, these problem-focused strategies are agreed to or partly agreed to in only $21.9 \%$ and $11.7 \%$ of the patients, respectively (data not shown). Problem-focused strategies may be less important and further investigations with more aspects of coping are needed.

Because of the multi-dimensional characteristics of quality of life, many factors that can have an affect are not included in the study. For example, aside from olfaction, cognitive performance, depression, and self-ratings on health status are all significant modulators of quality of life in patients with olfactory loss ${ }^{(7)}$. This may be the reason for the small correlation coefficients between QOD-NS or QOD-PS scores and the studied factors. Nonetheless, the results here reveal that certain factors do have significant effects on QOD-NS or QOD-PS (Table 1), and an inverse correlation exists between QOD-NS and QOD-PS scores.

\section{CONCLUSIONS}

The olfactory impact on quality of life is more significantly felt by patients younger than 33 years with poorer olfaction. It takes time to develop coping ability such that patients with longer disease duration and older age have increased 
emotional ability to cope. Emotional coping ability correlates significantly with decreasing olfactory impact on quality of life. It will be helpful for the patients to develop emotional coping early, especially if their olfactory function cannot be restored.

\section{ACKNOWLEDGEMENTS}

The study was support by a grant from Taipei Veterans General Hospital (Grant V99-C1-148).

\section{CONFLICT OF INTEREST}

The authors declare no conflicts of interest related to this study.

\section{REFERENCES}

1. Miwa T, Furukawa M, Tsukatani T, et al. Impact of olfactory impairment on quality of life and disability. Arch Otolaryngol Head Neck Surg. 2001; 127: 497-503.

2. Temmel AF, Quint C, Schickinger-Fischer B, et al. Characteristics of olfactory disorders in relation to major causes of olfactory loss. Arch Otolaryngol Head Neck Surg. 2002; 128: 635-641.

3. Frasnelli J, Hummel T. Olfactory dysfunction and daily life. Eur Arch Otorhinolaryngol. 2005; 262: 231-235.

4. Welge-Luessen A, Hummel T, Stojan T, Wolfensberger M. What is the correlation between ratings and measures of olfactory function in patients with olfactory loss? Am J Rhinol. 2005; 19: 567-571.

5. Nordin S, Bramerson A, Murphy C, Bende M. A Scandinavian adaptation of the Multi-Clinic Smell and Taste Questionnaire: evaluation of questions about olfaction. Acta Otolaryngol. 2003; 123: 536-542.

6. Bramerson A, Nordin S, Bende M. Clinical experience with patients with olfactory complaints, and their quality of life. Acta Otolaryngol. 2007; 127: 167-174.

7. Seo HS, Jeon KJ, Hummel T, Min BC. Influences of olfactory impairment on depression, cognitive performance, and quality of life in Korean elderly. Eur Arch Otorhinolaryngol. 2009; 266: 1739-1745.
8. Reden J, Mueller A, Mueller C, et al. Recovery of olfactory function following closed head injury or infections of the upper respiratory tract. Arch Otolaryngol Head Neck Surg. 2006; 132: 265-269.

9. London B, Nabet B, Fisher AR, et al. Predictors of prognosis in patients with olfactory disturbance. Ann Neurol. 2008; 63: 159-166.

10. Blomqvist EH, Bramerson A, Stjarne P, Nordin S. Consequences of olfactory loss and adopted coping strategies. Rhinology. 2004; 42: 189-194.

11. Hummel T, Sekinger B, Wolf SR, Pauli E, Kobal G. 'Sniffin' sticks': olfactory performance assessed by the combined testing of odor identification, odor discrimination and olfactory threshold. Chem Senses. 1997; 22: 39-52.

12. Shu CH, Yuan BC, Lin SH, Lin CZ. Cross-cultural application of the "Sniffin' Sticks" odor identification test. Am J Rhinol. 2007; 21: 570-573.

13. Yuan BC, Lee PL, Lee YL, Lin SH, Shu CH. Investigation of the Sniffin' Sticks olfactory test in Taiwan and comparison with different continents. J Chin Med Assoc. 2010; 73: 483-486.

14. Shu CH, Hummel T, Lee PL, et al. The proportion of self-rated olfactory dysfunction does not change across the life span. Am J Rhinol Allergy. 2009; 23: 413-416.

15. Landis BN, Hummel T, Hugentobler M, Giger R, Lacroix JS. Ratings of overall olfactory function. Chem Senses. 2003; 28: 691-694.

Chih-Hung Shu, MD

Department of Otolaryngology

Taipei Veterans General Hospital

No. 201, Sec. 2, Shipai Rd.

Beitou District

Taipei 11217

Taiwan

Tel: +886-2-2875 7337

Fax: +886-2-2875 7338

E-mail: chshu@vghtpe.gov.tw

\section{ERRATUM}

In the publication: "Sinonasal inverted papilloma: 84 patients treated by endoscopy and proposal for a new classification" by A. Dragonetti, R. Gera, A. Sciuto, A. Scotti, A. Bigoni, E. Barbaro, A. Minni (Rhinology 49: 206-212, 2011), the initial of the sixth author is cited incorrectly. The proper spelling should be: M. Barbaro. This erratum is to indicate that fact. 